\title{
Global Synchronization of Reaction-Diffusion Fractional-Order Memristive Neural Networks with Time Delay and Unknown Parameters
}

\author{
Wenjiao Sun, Guojian Ren $\left(\mathbb{D}\right.$, Yongguang Yu $\mathbb{D}^{D}$, and Xudong Hai \\ Department of Mathematics, Beijing Jiaotong University, Beijing 100044, China \\ Correspondence should be addressed to Yongguang Yu; ygyu@bjtu.edu.cn
}

Received 18 October 2019; Revised 3 January 2020; Accepted 1 April 2020; Published 31 May 2020

Academic Editor: Hiroki Sayama

Copyright (c) 2020 Wenjiao Sun et al. This is an open access article distributed under the Creative Commons Attribution License, which permits unrestricted use, distribution, and reproduction in any medium, provided the original work is properly cited.

\begin{abstract}
This paper investigated the global synchronization of fractional-order memristive neural networks (FMNNs). To deal with the effect of reaction-diffusion and time delay, fractional partial and comparison theorem are introduced. Based on the set value mapping theory and Filippov solution, the activation function is extended to discontinuous case. Adaptive controllers with a compensator are designed owing to the existence of unknown parameters, with the help of Gronwall-Bellman inequality. Numerical simulation examples demonstrate the availability of the theoretical results.
\end{abstract}

\section{Introduction}

As a generalization of differential and integral calculus from an integer order to arbitrary order, fractional calculus has more than 300 years history [1]. Since Mandelbort proposed the fact that lots of fractals exist in the field of technology, a whole new array of possibilities for natural science research has been opened [2]. Compared with the conventional integer order calculus, it shows some superiority in the aspect of memory and heredity [3]. That caused its wide range of applications [4-6].

Neural networks model, as we know, has great potential for controlling highly nonlinear and severe uncertain systems. Due to the ability of studying arbitrary continuous nonlinear functions, it has been fruitfully applied to optimization, secure communication, cryptography, and so on. Taking into account these facts, the combination of fractional calculus and neural networks model is a remarkably great improvement. This kind of model called fractionalorder neural networks (FNNs) was proposed by Arena in 1998 [7]. Afterwards, a myriad of studies have focused on the dynamical behaviors of FNNs. One of the most interesting issues is to explore the synchronization problem of FNNs. Various definition types of synchronization have been built
[8-10]. Among them, global synchronization is the most basic and widely applied one, since it guarantees the convergence of the system. From the perspective of engineering applications, it has achieved the required realistic standards $[11,12]$. Thus, global synchronization of FNNs has been extensively investigated to date [13-15].

Another hot topic of research is memristor-based neural networks, which were first put forward by Chua [16]. In 2008, a nanoscale memory was made by Williams group [17], which was named memristor. Memory characteristics and nanometer dimensions make the memristor show properties the same as the neurons in the human brain. From this standpoint, fractional-order memristive neural networks (FMNNs) have been suggested replacing the FNNs to emulate the human brain. Therefore, the analysis of FMNNs has become increasingly attractive to researchers. At present, there are many excellent results on synchronization of FMNNs [18-21]. Velmurugan investigated finitetime synchronization of FMNNs with the method of Laplace transform and generalized Gronwalls inequality [18]. The lag complete synchronization criteria of FMNNs are proposed by period intermittent control [19]. Delay-independent synchronization criteria for FMNNs are established by using the maximum modulus principle, the spectral radii of 
matrix, and Kakutani fixed point theorem [20]. Chen et al. designed a stabilizing state-feedback controller by employing the FO Razumikhin theorem and linear matrix inequalities [21]. Besides the abovementioned methods, adaptive mechanism has been proposed as a more effective strategy [22]. Compared with the conventional feedback control, the parameters of adaptive controllers will be updated automatically, which makes the energy be saved [23]. It also has significant effect on the analysis of fractional order nonlinear system, especially when the system has unknown parameters [24-27]. Thus, it is a natural idea to investigate the synchronization of FMNNs using adaptive control scheme.

It is a remarkable fact that time delays emerge in the communication of neurons [28, 29]. Furthermore, the occurrence of diffusion phenomena has a significant impact in the electrons' transportation through a nonuniform electromagnetic field. That means, not only the time but also their spatial positions affect the dynamic behaviors of networks. The models covering time delay and reaction-diffusion are good mimicry for the real neural networks in terms of application, but the existence of time delays and reaction-diffusion could bring about some undesirable behaviors [30,31]. Drawing on the abovementioned concerns, special attention should be paid on time delay and reactiondiffusion. However, the research on FMNNs with both reaction-diffusion and time delay is still in its infancy $[32,33]$.

For realistic neural networks systems, one must include uncertainties. Uncertainty could occur in parameters in the mathematical model, for they are hardly to be exactly known in advance [34-36]. Therefore, the study on synchronization of the drive-response system considering unknown parameters is one of the most challenging tasks. Expanding on the existing literature, the global synchronization problem for reaction-diffusion FMNNs with time delay and unknown parameters is proposed firstly. The main contents of this paper are the following. (1) Based on fractional comparison theorem, the problem of synchronization for the integral delayed neural networks is generalized to the fractional order case. (2) By utilizing Caputo partial fractional derivative, reaction-diffusion is emphasized for the FMNNs, which extends the problem from the time domain to timespatial dimension. (3) With the help of functional differential inclusions, the solution of the discontinuous systems is guaranteed in the sense of Filippov. (4) Employing Gronwall-Bellman inequality, the adaptive controllers with compensator are designed to achieve the synchronization, despite the presence of parametric uncertainties.

This paper is structured as follows. In Section 2, we briefly introduce preliminaries and model description. Two types of adaptive controllers for global synchronization of the drive-response system are designed in Section 3. In Section 4, two simulations are shown to verify the correctness of the proposed results. A short conclusion and some discussions are given in Section 5.

Notations. let $\mathbb{R}^{n}$ be the space of $n$-dimensional Euclidean space. The norm of vector $x=\left(x_{1}, x_{2}, \ldots, x_{n}\right)^{T} \in \mathbb{R}^{n}$ is defined as $\|x\|=\left(\sum_{q=1}^{n} x_{q}^{2}\right)^{1 / 2}$. A bounded open-set $\Omega \subset \mathbb{R}^{n}$ containing the origin equips with the smooth boundary $\partial \Omega$ and mes $\Omega>0$.

\section{Preliminaries and Model Description}

Preliminaries about fractional calculus and the model of reaction-diffusion FMNNs with time delay are introduced in this section. Concept of Filippov solution, fractional comparison theorem, and Gronwall-Bellman inequality need to be emphasized.

2.1. Preliminaries. Definition and the properties about Caputo Riemann-Liuville fractional calculus are given in the following.

Definition 1 (see [37]). For any $t>0$, the time Caputo fractional derivative of order $\alpha(0<\alpha<1)$ for a function $f(t, x) \in C^{1}[[0, b] \times \Omega, \mathbb{R}]$ is defined by

$$
\frac{\partial^{\alpha} f(t, x)}{\partial t^{\alpha}}=\frac{1}{\Gamma(1-\alpha)} \int_{0}^{t} \frac{\partial f(s, x)}{\partial s} \frac{\mathrm{d} s}{(t-s)^{\alpha}},
$$

where $\quad \Gamma(z)=\int_{0}^{\infty} e^{-t} t^{z-1} \mathrm{~d} t . \quad$ Particularly, when $f(t, \cdot)=f(t)$, then

$$
\frac{\partial^{\alpha} f(t, \cdot)}{\partial t^{\alpha}}=\frac{\mathrm{d}^{\alpha} f(t)}{\mathrm{d} t^{\alpha}}={ }_{0}^{C} D_{t}^{\alpha} f(t) .
$$

It should be noted that the initial conditions of Caputo fractional derivative are same as integer-order derivative, which makes Caputo fractional derivative equip specific physical sense in the real world. In this paper, simple notation $D^{\alpha}$ is used to replace ${ }_{0}^{C} D_{t}^{\alpha}$.

Definition 2 (see [37]). Riemann-Liuville fractional integral of order $\alpha$ for a function $f(t):([0,+\infty) \longrightarrow R)$ is defined by

$$
{ }_{R}^{0} I_{t}^{\alpha} f(t)=\frac{1}{\Gamma(\alpha)} \int_{0}^{t} \frac{f(\tau)}{(t-\tau)^{1-\alpha}} \mathrm{d} \tau,
$$

where $0<\alpha<1$.

Proposition 1 (see [37]). For arbitrary constants $v_{1}$ and $v_{2}$, there is

$$
D^{\alpha}\left(v_{1} f(t)+v_{2} g(t)\right)=v_{1} D^{\alpha} f(t)+v_{2} D^{\alpha} g(t) .
$$

Proposition 2 (see [37]). If $0<\alpha<1$ and $f(t) \in C^{1}[0, t]$, then ${ }_{R}^{0} I_{t}^{\alpha} D^{\alpha} f(t)=f(t)-f(0)$.

Several important conclusions about the fractional-order system are listed below.

Lemma 1 (see [33]). Let $f(t, x):[0, b] \times \Omega \longrightarrow \mathbb{R}$, be $a$ continuous and differentiable function on $t$; then, for any $t \geq 0, x \in \Omega$,

$$
\frac{1}{2} \frac{\partial^{\alpha} f^{2}(t, x)}{\partial t^{\alpha}} \leq f(t, x) \frac{\partial^{\alpha} f(t, x)}{\partial t^{\alpha}},
$$

where $0<\alpha<1$. Particularly, when $\alpha=1$, there is 


$$
\frac{1}{2} \frac{\partial f^{2}(t, x)}{\partial t} \leq f(t, x) \frac{\partial f(t, x)}{\partial t}
$$

Lemma 2 (see [38]). Consider the system as follows:

$$
\left\{\begin{array}{l}
D^{\alpha} V(x(t))=-\rho V(x(t))+k V(x(t-\tau)), \\
x(t)=\phi(t) \\
t \in[-\tau, 0]
\end{array}\right.
$$

where $\rho, k \geq 0$ and $V(x) \geq 0$. The solution of the system is asymptotically stable, when $k<\rho \sin (\alpha \pi / 2)$.

Lemma 3 (fractional comparison theorem; see [38]). Consider the following fractional order inequality:

$$
\left\{\begin{array}{l}
D^{\alpha} V(x(t)) \leq-\rho V(x(t))+k V(x(t-\tau)), \\
V(x(t))=h(t), t \in[-\tau, 0]
\end{array}\right.
$$

and fractional order system:

$$
\left\{\begin{array}{l}
D^{\alpha} V(y(t))=-\rho V(y(t))+k V(y(t-\tau)), \\
V(y(t))=h(t), t \in[-\tau, 0]
\end{array}\right.
$$

where $\alpha \in(0,1), \tau>0, x(t), y(t) \in R^{n}$, and $V(x)$ and $V(y)$ are continuous and nonnegative functions in $(0,+\infty)$, $h(t) \geq 0$ on $[-\tau, 0]$. If $\rho>0$ and $k>0$, then the inequality holds:

$$
V(x(t)) \leq V(y(t))
$$

for all $t \in[0,+\infty)$.

Lemma 4 (see [37, 39]). Suppose that e $(x, t): \Omega \times R^{+} \longrightarrow R$ is integrable on $\Omega$ and is derivable respect to $t$. Denote $V(t)=\int_{\Omega} e(x, t) d x$, then

$$
D^{\alpha} V(t)=\int_{\Omega} D^{\alpha} e(x, t) \mathrm{d} x .
$$

Lemma 5 (see [40]). If $x(t), y(t) \in C^{1}\left[t_{0}, b\right]$ with $x(t) \leq y(t)$, then $\forall t \in\left[t_{0}, b\right]$ there is ${ }_{t_{0}}^{R} I_{t}^{\alpha} x(t) \leq{ }_{t_{0}}^{R} I_{t}^{\alpha} y(t)$.

Lemma 6 (see [41]). Let $\Omega$ be a cube $\left|x_{q}\right|<l_{q}, q=1,2, \ldots, n$, and let $f(x)$ be a real-valued function belonging to $C^{1}(\Omega)$ which vanishes on the boundary of the $\Omega$, i.e., $\left.f(x)\right|_{\partial \Omega}=0$, then

$$
\int_{\Omega} f^{2}(x) \mathrm{d} x \leq l_{q}^{2} \int_{\Omega}\left|\frac{\partial f(x)}{\partial x_{q}}\right|^{2} \mathrm{~d} x
$$

Lemma 7 (Gronwall-Bellman inequality; see [42]). If $z(t)$ satisfies $z(t) \leq \int_{0}^{t} a(\tau) z(\tau) d \tau+b(t)$, where $a(t)$ and $b(t)$ are the known real functions, then

$$
z(t) \leq b(0) \exp \left(\int_{0}^{t} a(\tau) \mathrm{d} \tau\right)+\int_{0}^{t} \dot{b}(\tau) \exp \left(\int_{\tau}^{t} a(r) \mathrm{d} r\right) \mathrm{d} \tau \text {. }
$$

If $b(t)$ is a constant,

$$
z(t) \leq b(0) \exp \left(\int_{0}^{t} a(\tau) \mathrm{d} \tau\right)
$$

2.2. System Description. Reaction-diffusion FMNNs with time delay are described as

$$
\begin{aligned}
\frac{\partial^{\alpha} u_{i}(t, x)}{\partial t^{\alpha}}= & \sum_{q=1}^{n} d_{i q} \Delta u_{i}(t, x)-c_{i} u_{i}(t, x)+\sum_{j=1}^{m}\left\langle a_{i j} f_{j}\right\rangle(t, x) \\
& +\sum_{j=1}^{m}\left\langle b_{i j} g_{j}\right\rangle(t-\tau, x)+I_{i},
\end{aligned}
$$

where $i=1,2, \ldots, m, m \geq 2$ is the quantity of units; $0<\alpha<1$ refers to the order of the equation; $u_{i}(t, x)$ represents the state of the $i$ th unit at time $t$ and in space $x ; t \in J=[0, T]$ represents the time variable; $\Delta$ is a Laplace operator, which means $\Delta u_{i}(t, x)=\partial^{2} u_{i}(t, x) / \partial x_{q}^{2}$; the smooth constants $d_{i q} \geq 0$ work as the transmission diffusion operator along the $i$ th unit at $q$ th position; $\left\langle a_{i j} f_{j}\right\rangle(t, x)=a_{i j}\left(u_{i}(t, x)\right)$ $f_{j}\left(u_{j}(t, x)\right), \quad\left\langle b_{i j} g_{j}\right\rangle(t-\tau, x)=b_{i j}\left(u_{i}(t, x)\right) g_{j}\left(u_{j}(t-\tau\right.$, $x)$ ), where $a_{i j}\left(u_{i}(t, x)\right)$ and $b_{i j}\left(u_{i}(t, x)\right)$ stand for the strength of the $j$ th unit on the $i$ th unit at time $t$ in space $x$ and at time $t-\tau$ in space $x$, respectively; $c_{i}>0$ is the selffeedback connection weight; $f_{j}(\cdot)$ and $g_{j}(\cdot)$ denote the discontinuous activation functions; $I_{i}$ signifies the external input; and $\tau \geq 0$ stands for time delay. In addition, the memristor-based connection weights $a_{i j}\left(u_{i}(t\right.$, $x))$ and $b_{i j}\left(u_{i}(t, x)\right)$ are given by

$$
\begin{aligned}
& a_{i j}\left(u_{i}(t, x)\right)= \begin{cases}\widehat{a}_{i j}, & \left|u_{i}(t, \cdot)\right|>T_{j}^{a}, \\
\text { unsureness, } & \left|u_{i}(t, \cdot)\right|=T_{j}^{a}, \\
\check{a}_{i j}, & \left|u_{i}(t, \cdot)\right|<T_{j}^{a},\end{cases} \\
& b_{i j}\left(u_{i}(t, x)\right)= \begin{cases}\widehat{b}_{i j}, & \left|u_{i}(t, \cdot)\right|>T_{j}^{b}, \\
\text { unsureness, } & \left|u_{i}(t, \cdot)\right|=T_{j}^{b}, \\
\check{b}_{i j}, & \left|u_{i}(t, \cdot)\right|<T_{j}^{b},\end{cases}
\end{aligned}
$$

where $i, j=1,2, \ldots, m, t \geq 0$, switching jumps $T_{j}^{a}, T_{j}^{b}>0$ and $\widehat{a}_{i j}, \check{a}_{i j}, \widehat{b}_{i j}$, and $\check{b}_{i j}$ are all constants.

Consider the following Dirichlet-type boundary conditions of (15)

$$
u_{i}(t, x)=0, \quad t \in[-\tau, \infty), x \in \partial \Omega,
$$

and initial conditions

$$
u_{i}(s, x)=\varphi_{0 i}(s, x), \quad s \in[-\tau, 0], x \in \Omega,
$$

where $\varphi_{0}=\left(\varphi_{01}, \varphi_{02}, \ldots, \varphi_{0 m}\right)^{T}$ are real-value continuous functions defined on $[-\tau, 0] \times \Omega$.

Take (15) as the drive system, then the corresponding response system is defined by 


$$
\begin{aligned}
\frac{\partial^{\alpha} v_{i}(t, x)}{\partial t^{\alpha}}= & \sum_{q=1}^{n} d_{i q} \Delta v_{i}(t, x)-c_{i} v_{i}(t, x)+\sum_{j=1}^{m}\left\langle a_{i j} f_{j}\right\rangle(t, x) \\
& +\sum_{j=1}^{m}\left\langle b_{i j} g_{j}\right\rangle(t-\tau, x)+I_{i}+\kappa_{i}(t, x),
\end{aligned}
$$

where $i=1,2, \ldots, m$ and $\kappa_{i}(t, x)$ is the controller which will be designed later.

Similarly, there are

$$
\begin{aligned}
& v_{i}(t, x)=0, \quad t \in[-\tau, \infty), x \in \partial \Omega, \\
& v_{i}(s, x)=\Phi_{0 i}(s, x), \quad s \in[-\tau, 0], x \in \Omega,
\end{aligned}
$$

and $\Phi_{0}=\left(\Phi_{01}, \Phi_{02}, \ldots, \Phi_{0 m}\right)^{T}$, which have the same property to $\varphi_{0}$.

Since the right-hand sides of equations are discontinuous, the solutions of the equations in the conventional sense have been shown to be invalid. In this case, Filippov solutions [43] are introduced to deal with the discontinuous equations in functional differential inclusions framework. The solution's global existence for fractional differential inclusions with time delay is given by $[44]$.

Assumption 1. For each $i \in \mathbb{N}, f_{i}(\cdot): \mathbb{R} \longrightarrow \mathbb{R}$ is piecewise continuous function. Moreover, $f_{i}(\cdot)$ has at most limited the number of discontinuous points on any compact interval of $\mathbb{R}$. The abovementioned properties are the same to $g_{i}(\cdot)$. Based on the definition of Filippov solution, (15) can be rewritten as

$$
\begin{aligned}
\frac{\partial u_{i}(t, x)}{\partial t^{\alpha}} \in & \sum_{q=1}^{n} d_{i q} \Delta u_{i}(t, x)-c_{i} u_{i}(t, x) \\
& +\sum_{j=1}^{m} K\left[a_{i j}\right](t) \overline{\operatorname{co}}\left[f_{j}\left(u_{j}(t, x)\right)\right] \\
& +\sum_{j=1}^{m} K\left[b_{i j}\right](t) \overline{\operatorname{co}}\left[g_{j}\left(u_{j}(t-\tau, x)\right)\right]+I_{i},
\end{aligned}
$$

where $i=1,2, \ldots, m, u_{i}(t, x)$ are absolutely continuous on any compact subinterval of $\left[t_{0}, \infty\right) \times \Omega$ :

$$
\begin{aligned}
& K\left[a_{i j}\right](t)= \begin{cases}\hat{a}_{i j}, & u_{j}(t, \cdot)>\left|T_{j}^{a}\right|, \\
\overline{\operatorname{co}}\left\{\widehat{a}_{i j}, \check{a}_{i j}\right\}, & u_{j}(t, \cdot)=\left|T_{j}^{a}\right|, \\
\check{a}_{i j}, & u_{j}(t, \cdot)<\left|T_{j}^{a}\right|,\end{cases} \\
& K\left[b_{i j}\right](t)= \begin{cases}\widehat{b}_{i j}, & u_{j}(t, \cdot)>\left|T_{j}^{b}\right|, \\
\overline{\operatorname{co}}\left\{\widehat{b}_{i j}, \check{b}_{i j}\right\}, & u_{j}(t, \cdot)=\left|T_{j}^{b}\right|, \\
\check{b}_{i j}, & u_{j}(t, \cdot)<\left|T_{j}^{b}\right| .\end{cases}
\end{aligned}
$$

Or equivalently, there are measurable functions $\tilde{a}_{i j}(\cdot) \in K\left[a_{i j}\right](\cdot), \widetilde{b}_{i j}(\cdot) \in K\left[b_{i j}\right](\cdot), \widetilde{f}_{1 j}(\cdot) \in \overline{\mathrm{co}}\left[f_{j}\left(u_{j}(\cdot)\right)\right]$, and $\tilde{g}_{1 j}(\cdot) \in \overline{\mathrm{co}}\left[g_{j}\left(u_{j}(\cdot)\right)\right]$ satisfy

$$
\begin{aligned}
\frac{\partial^{\alpha} u_{i}(t, x)}{\partial t^{\alpha}}= & \sum_{q=1}^{n} d_{i q} \Delta u_{i}(t, x)-c_{i} u_{i}(t, x)+\sum_{j=1}^{m} \widetilde{a}_{i j}(t) \widetilde{f}_{1 j}(t, x) \\
& +\sum_{j=1}^{m} \widetilde{b}_{i j}(t) \widetilde{g}_{1 j}(t-\tau, x)+I_{i},
\end{aligned}
$$

for a.e. $t \in\left[t_{0}, \infty\right), x \in \Omega, i=1,2, \ldots, m$.

For writing convenience, we denote $\max \left\{||_{i j}|,| \breve{a}_{i j} \mid\right\}$ as $a_{i j}^{\max }$ and $\max \left\{\left|\widehat{b}_{i j}\right|,\left|\check{b}_{i j}\right|\right\}$ as $b_{i j}^{\max }$.

The IVP of the driven system (15) can be written as

$$
\left\{\begin{array}{l}
\frac{\partial^{\alpha} u_{i}(t, x)}{\partial t^{\alpha}}=\sum_{q=1}^{n} d_{i q} \Delta u_{i}(t, x)-c_{i} u_{i}(t, x)+\sum_{j=1}^{m}\left\langle\widetilde{a}_{i j}, \tilde{f}_{1 j}\right\rangle(t, x) \\
+\sum_{j=1}^{m}\left\langle\tilde{b}_{i j}, \widetilde{g}_{1 j}\right\rangle(t-\tau, x)+I_{i}, \\
u_{i}(t, x)=0, \quad t \in[-\tau, \infty), x \in \partial \Omega, \\
u_{i}(s, x)=\varphi_{0 i}(s, x), \quad t \in[-\tau, 0], x \in \Omega, \\
\tilde{f}_{1 j}(s, x)=\gamma_{j}(s, x), \quad \text { a.e. } t \in[-\tau, 0], x \in \Omega, \\
\tilde{g}_{1 j}(s, x)=\eta_{j}(s, x), \quad \text { a.e. } t \in[-\tau, 0], x \in \Omega .
\end{array}\right.
$$

The IVP of the response system (19) under the controller could be given by

$$
\begin{cases}\frac{\partial^{\alpha} v_{i}(t, x)}{\partial t^{\alpha}}=\sum_{q=1}^{n} d_{i q} \Delta v_{i}(t, x)-c_{i} v_{i}(t, x)+\sum_{j=1}^{m}\left\langle\widetilde{a}_{i j}, \widetilde{f}_{2 j}\right\rangle(t, x) \\ +\sum_{j=1}^{m}\left\langle\widetilde{b}_{i j}, \widetilde{g}_{2 j}\right\rangle(t-\tau, x)+I_{i}+\kappa_{i}(t, x), \\ v_{i}(t, x)=0, \quad t \in[-\tau, \infty), x \in \partial \Omega, \\ v_{i}(s, x)=\Phi_{0 i}(s, x), \quad t \in[-\tau, 0], x \in \Omega, \\ \widetilde{f}_{2 j}(s, x)=\xi_{j}(s, x), \quad \text { a.e. } t \in[-\tau, 0], x \in \Omega, \\ \tilde{g}_{2 j}(s, x)=\omega_{j}(s, x), & \text { a.e. } t \in[-\tau, 0], x \in \Omega,\end{cases}
$$

where $i, j=1,2, \ldots, m . \quad\left\langle\widetilde{a}_{i j}, \widetilde{f}_{1 j}\right\rangle(t, x)=\widetilde{a}_{i j}(t) \tilde{f}_{1 j}(t, x)$, $\left\langle\widetilde{b}_{i j}, \tilde{g}_{1 j}\right\rangle(t-\tau, x)=\widetilde{b}_{i j}(t) \widetilde{g}_{1 j}(t-\tau, x), \quad\left\langle\widetilde{a}_{i j}, \widetilde{f}_{2 j}\right\rangle(t, x)=$ $\tilde{a}_{i j}(t) \widetilde{f}_{2 j}(t, x)$, and $\left\langle\widetilde{b}_{i j}, \tilde{g}_{2 j}\right\rangle(t-\tau, x)=\widetilde{b}_{i j}(t) \widetilde{g}_{2 j}(t-\tau, x)$, $\tilde{f}_{2 j}(\cdot) \in \overline{\mathrm{co}}\left[f_{j}\left(v_{j}(\cdot)\right)\right]$ and $\tilde{g}_{2 j}(\cdot) \in \overline{\mathrm{co}}\left[g_{j}\left(v_{j}(\cdot)\right)\right]$. 
Now, let $e_{i}(t, x)=u_{i}(t, x)-v_{i}(t, x)$ be the synchronization error between (15) and (19). The IVP about the error system is of the form

$$
\left\{\begin{array}{l}
\frac{\partial^{\alpha} e_{i}(t, x)}{\partial t^{\alpha}}=\sum_{q=1}^{n} d_{i q} \Delta e_{i}(t, x)-c_{i} e_{i}(t, x)+\sum_{j=1}^{m}\left\langle\widetilde{a}_{i j}, \bar{f}_{j}\right\rangle(t, x) \\
+\sum_{j=1}^{m}\left\langle b_{i j}, \bar{g}_{j}\right\rangle(t-\tau, x)+\kappa_{i}(t, x), \\
e_{i}(t, x)=0, \quad t \in[-\tau, \infty), x \in \partial \Omega, \\
e_{i}(s, x)=\bar{\varphi}_{0 i}(s, x), \quad s \in[-\tau, 0], x \in \Omega,
\end{array}\right.
$$

where $i, j=1,2, \ldots, m$, the symbol $\left\langle\tilde{a}_{i j}, \bar{f}_{j}\right\rangle(t, x)$ is written as $\widetilde{a}_{i j}(t)\left[\tilde{f}_{2 j}(t, x)-\widetilde{f}_{1 j}(t, x)\right]$ and $\left\langle b_{i j}, \bar{g}_{j}\right\rangle(t-\tau, x)$ which stands for $\widetilde{b}_{i j}(t)\left[\widetilde{g}_{2 j}(t-\tau, x)-\widetilde{g}_{1 j}(t-\tau, x)\right]$.

Denote $\left\|e_{i}(t, x)\right\|=\left[\int_{\Omega} \sum_{i=1}^{m} e_{i}^{2}(t, x) \mathrm{d} x\right]^{1 / 2}$ as the norm of vector $e(t, x)=\left(e_{1}(t, x), e_{2}(t, x), \ldots, e_{m}(t, x)\right)^{\mathrm{T}} \in R^{m}$.

Definition 3. The drive system (15) and response system (19) is said to be globally synchronized, if $\lim _{t \longrightarrow \infty}\left\|e_{i}(t, x)\right\|=0$.

Remark 1. Owing to the zero Dirichlet boundary conditions of (24) and (25), a zero equilibrium state of error system (26) exists. The global synchronization of systems (24) and (25) corresponds to the global asymptotic stability of the zero equilibrium of system (26).

\section{Main Results}

The abovementioned synchronization problem in Section 2 are considered in two cases, namely, the drive system (15) has known or unknown parameters.

3.1. Global Synchronization for Systems with Known Parameters. First of all, we assume that the parameters in (15) are known. Then, the following adaptive controllers are proposed to implement global synchronization.

Assumption 2. For $i=1,2, \ldots, m$, there are positive constants $p_{i}$ and $q_{i}$ such that

$$
\begin{aligned}
& \sup _{\alpha_{i} \in \overline{\mathrm{co}}\left[f_{i}(\mu)\right], \beta_{i} \in \overline{\mathrm{co}}\left[f_{i}(\vartheta)\right]}\left|\alpha_{i}-\beta_{i}\right| \leq p_{i}\left|\mu_{i}-\vartheta_{i}\right|+q_{i}, \quad \forall \mu, \vartheta \in \mathbb{R}, \\
& \sup _{\alpha_{i} \in \overline{\mathrm{co}}\left[g_{i}(\mu)\right], \beta_{i} \in \overline{\operatorname{co}}\left[g_{i}(\vartheta)\right]}\left|\alpha_{i}-\beta_{i}\right| \leq r_{i}\left|\mu_{i}-\vartheta_{i}\right|+s_{i}, \quad \forall \mu, \vartheta \in \mathbb{R},
\end{aligned}
$$

where for any $\theta \in \mathbb{R}$,

$$
\begin{aligned}
& \overline{\mathrm{co}}\left[f_{i}(\theta)\right]=\left[\min \left\{f_{j}^{-}(\theta), f_{j}^{+}(\theta)\right\}, \max \left\{f_{j}^{-}(\theta), f_{j}^{+}(\theta)\right\}\right], \\
& \overline{\mathrm{co}}\left[g_{i}(\theta)\right]=\left[\min \left\{g_{j}^{-}(\theta), g_{j}^{+}(\theta)\right\}, \max \left\{g_{j}^{-}(\theta), g_{j}^{+}(\theta)\right\}\right] .
\end{aligned}
$$

For the convenience of description, the following notations are introduced:

$$
\begin{aligned}
\sigma_{i} & =2\left[-c_{i}+\sum_{q=1}^{n}\left(-\frac{d_{i q}}{l_{q}^{2}}\right)\right]+\sum_{j=1}^{m}\left(a_{i j}^{\max } p_{j}+a_{j i}^{\max } p_{i}\right)+\sum_{j=1}^{m} b_{i j} r_{j}, \\
\sigma_{i}^{*} & =2\left(\sum_{j=1}^{m} a_{i j}^{\max } q_{j}+\sum_{j=1}^{m} b_{i j}^{\max } s_{j}\right), \\
\sigma_{i}^{* *} & =\sum_{j=1}^{m} b_{i j}^{\max } r_{i} \\
\bar{\sigma}_{i} & =-2 c_{i}+\sum_{j=1}^{m}\left(a_{i j}^{\max } p_{j}+a_{j i}^{\max } p_{i}+b_{i j} r_{j}\right), \\
\widetilde{\sigma}_{i} & =-2\left[c_{i}+\sum_{q=1}^{n}\left(\frac{d_{i q}}{l_{q}^{2}}\right)\right]+\sum_{j=1}^{m}\left(a_{i j}^{\max } p_{j}+a_{j i}^{\max } p_{i}\right), \\
\bar{\sigma}_{i}^{*} & =2 \sum_{j=1}^{m} a_{i j}^{\max } q_{j} .
\end{aligned}
$$

Theorem 1. Under Assumption 1 and Assumption 2, the global synchronization of (24) and (25) can be achieved based on the following adaptive controllers:

$$
\left\{\begin{array}{l}
\kappa_{i}(t, x)=-\left(\ell_{i}(t, x)+\bar{\ell}_{i}\right) e_{i}(t, x)-\omega_{i} \operatorname{sgn}\left(e_{i}(t, x)\right), \\
D^{\alpha} \ell_{i}(t, x)=\varpi_{i} e_{i}^{2}(t, x), \quad i=1,2, \ldots, m,
\end{array}\right.
$$

where $\ell_{i}(0, x) \geq 0, \Phi_{i}>0$, and $\bar{\ell}_{i}$ and $\omega_{i}$ are constants which satisfy the following inequalities:

$$
\begin{aligned}
& \bar{\ell}_{i}>\frac{1}{2}\left(\sigma_{i}+\sigma_{i}^{* *} \csc \left(\frac{\alpha \pi}{2}\right)\right)>0, \\
& \omega_{i}>\frac{1}{2} \sigma_{i}^{*} .
\end{aligned}
$$

Proof. Employ the following auxiliary function:

$$
V(t, e(t, \cdot))=\int_{\Omega} \frac{1}{2} \sum_{i=1}^{m} e_{i}^{2}(t, x) \mathrm{d} x .
$$

By the means of Lemma 1, the inequality can be established:

$$
\frac{\mathrm{d}^{\alpha}}{\mathrm{d} t^{\alpha}}\left(\int_{\Omega} e_{i}^{2}(t, x) \mathrm{d} x\right) \leq 2 \int_{\Omega} e_{i}(t, x) \frac{\partial^{\alpha} e_{i}(t, x)}{\partial t^{\alpha}} \mathrm{d} x .
$$

According to Lemma 4, taking the derivative of (32) yields 


$$
\begin{aligned}
D^{\alpha} V(t, e(t, \cdot)) \leq & \sum_{i=1}^{m} \int_{\Omega} e_{i}(t, x)\left[\sum_{q=1}^{n} d_{i q} \Delta e_{i}(t, x)\right]-c_{i} e_{i}(t, x) \\
& +\sum_{j=1}^{m}\left\langle\widetilde{a}_{i j}, \bar{f}_{j}\right\rangle(t, x)+\sum_{j=1}^{m}\left\langle b_{i j}, \bar{g}_{j}\right\rangle(t-\tau, x) \\
& +\kappa_{i}(t, x) \mathrm{d} x .
\end{aligned}
$$
that

Using Green's formula and Lemma 6, it can be obtained

$$
\sum_{q=1}^{n} \int_{\Omega} e_{i}(t, x) d_{i q} \Delta e_{i}(t, x) \mathrm{d} x \leq-\sum_{q=1}^{n} \int_{\Omega} \frac{d_{i q}}{l_{q}} e_{i}^{2}(t, x) \mathrm{d} x .
$$

Recalling Assumption 1 and Assumption 2, the inequalities hold:

$$
\begin{aligned}
& \sum_{j=1}^{m} \int_{\Omega} e_{i}(t, x)\left\langle\widetilde{a}_{i j}, \bar{f}_{j}\right\rangle(t, x) \mathrm{d} x \leq \frac{1}{2} \sum_{j=1}^{m} a_{i j}^{\max } p_{j} \int_{\Omega} e_{i}^{2}(t, x) \\
& \quad+e_{j}^{2}(t, x) \mathrm{d} x+\sum_{j=1}^{m} a_{i j}^{\max } q_{j} \int_{\Omega}\left|e_{i}(t, x)\right| \mathrm{d} x, \\
& \sum_{j=1}^{m} \int_{\Omega} e_{i}(t, x)\left\langle b_{i j}, \bar{g}_{j}\right\rangle(t-\tau, x) \mathrm{d} x \leq \frac{1}{2} \sum_{j=1}^{m} b_{i j}^{\max } r_{j} \int_{\Omega} e_{i}^{2}(t, x) \\
& \quad+e_{j}^{2}(t-\tau, x) \mathrm{d} x+\sum_{j=1}^{m} b_{i j}^{\max } s_{j} \int_{\Omega}\left|e_{i}(t, x)\right| \mathrm{d} x .
\end{aligned}
$$

Combining controller (30) designed in Theorem 1, it follows that

$$
\begin{aligned}
D^{\alpha} V(t, e(t, \cdot)) \leq & \frac{1}{2} \sum_{i=1}^{m} \sigma_{i} \int_{\Omega} e_{i}^{2}(t, x) \mathrm{d} x+\frac{1}{2} \sum_{i=1}^{m} \sigma_{i}^{* *} \\
& \int_{\Omega} e_{i}^{2}(t-\tau, x) \mathrm{d} x+\frac{1}{2} \sum_{i=1}^{m} \sigma_{i}^{*} \int_{\Omega}\left|e_{i}(t, x)\right| \mathrm{d} x \\
& -\sum_{i=1}^{m} \int_{\Omega}\left(l_{i}(t, x)+\bar{\ell}_{i}\right) e_{i}^{2}(t, x) \mathrm{d} x \\
& -\sum_{i=1}^{m} \omega_{i} \int_{\Omega}\left|e_{i}(t, x)\right| \mathrm{d} x .
\end{aligned}
$$

From Definition 2 and Property 2, one obtains

$$
\ell_{i}(t, x)=\ell_{i}(0, x)+\frac{1}{\Gamma(\alpha)} \int_{0}^{t}(t-\tau)^{\alpha} \varpi_{i} e_{i}^{2}(t, x) \mathrm{d} x
$$

Substituting (38) into (37) and noting that $\ell_{i}$ and $\omega_{i}$ meet inequality (31), it can be deduced that

$$
D^{\alpha} V(t, e(t, \cdot)) \leq-\lambda_{1} V(t, e(t, \cdot))+\rho_{1} V(t-\tau, e(t, \cdot)),
$$

where $\lambda_{1}=\sum_{i=1}^{m}\left(2 \bar{\ell}_{i}-\sigma_{i}\right)$ and $\rho_{1}=\sum_{i=1}^{m} \sigma_{i}^{* *}$.

According to Lemma 2 and Lemma 3, it can be assert that the global synchronization between (24) and (25) can be realized.

The proof of Theorem 1 completes.

Remark 2. When $\alpha=1$, the error system (26) be changed into the integer form. The conclusion is the same as the result in Theorem 3.1 in article [45]. If the reaction-diffusion in space $\Omega$ disappears, then the states of the system's variable are only relevant to time but independent of space. Thus, the error system (26) can be converted to

$$
\left\{\begin{array}{l}
D^{\alpha} e_{i}(t)=-c_{i} e_{i}(t)+\sum_{j=1}^{m}\left\langle\tilde{a}_{i j}, \bar{f}_{j}\right\rangle(t)+\sum_{j=1}^{m}\left\langle b_{i j}, \bar{g}_{j}\right\rangle(t-\tau)+\kappa_{i}(t), \\
e_{i}(s)=\bar{\varphi}_{0 i}(s), \quad s \in[-\tau, 0], \quad i=1,2, \ldots, m .
\end{array}\right.
$$

For convenience, the inequalities are given by

$$
\begin{aligned}
& \bar{\ell}_{i}>\frac{1}{2}\left(\bar{\sigma}_{i}+\sigma_{i}^{* *} \csc \left(\frac{\alpha \pi}{2}\right)\right)>0, \\
& \omega_{i}>\frac{1}{2} \sigma_{i}^{*} .
\end{aligned}
$$

Corollary 1. Under Assumption 1 and Assumption 2, the global synchronization between (24) and (25) can be achieved based on the following adaptive controllers:

$$
\left\{\begin{array}{l}
\kappa_{i}(t)=-\left(\ell_{i}(t)+\bar{\ell}_{i}\right) e_{i}(t)-\omega_{i} \operatorname{sgn}\left(e_{i}(t)\right), \\
D^{\alpha} \ell_{i}(t)=\omega_{i} e_{i}^{2}(t), i=1,2, \ldots, m
\end{array}\right.
$$

where $\ell_{i}(0)>0, \omega_{i}>0$, and $\bar{\ell}_{i}$ and $\omega_{i}$ are constants which satisfy inequality (41).

Remark 3. In fact, synchronization of FMNNs with time delay has been investigated in [20]. The results about synchronization of the systems with single delay in Theorem 3 in [20] are the same as Corollary 1 obtained in this paper. Suppose that there is no time delay $\tau$ in the course of communication between the different neurons; then, error system (26) can be reduced to

$$
\left\{\begin{array}{l}
\frac{\partial^{\alpha} e_{i}(t, x)}{\partial t^{\alpha}}=\sum_{q=1}^{n} d_{i q} \Delta e_{i}(t, x)-c_{i} e_{i}(t, x)+\sum_{j=1}^{m}\left\langle\widetilde{a}_{i j}, \bar{f}_{j}\right\rangle \\
(t, x)+\kappa_{i}(t, x), \\
e_{i}(t, x)=0, \quad t \in[0, \infty), x \in \partial \Omega, \\
e_{i}(s, x)=\bar{\varphi}_{0 i}(s, x), \quad s=0, x \in \Omega .
\end{array}\right.
$$


For convenience, the inequalities are given by

$$
\begin{aligned}
& \bar{\ell}_{i}>\frac{1}{2} \widetilde{\sigma}_{i}>0, \\
& \omega_{i}>\frac{1}{2} \bar{\sigma}_{i}^{*} .
\end{aligned}
$$

Corollary 2. Under Assumption 1 and Assumption 2, the global synchronization between (24) and (25) can be realized based on the following adaptive controllers:

$$
\left\{\begin{array}{l}
\kappa_{i}(t, x)=-\left(\ell_{i}(t, x)+\bar{\ell}_{i}\right) e_{i}(t, x)-\omega_{i} \operatorname{sgn}\left(e_{i}(t, x)\right), \\
D^{\alpha} \ell_{i}(t, x)=\varpi_{i} e_{i}^{2}(t, x), \\
i=1,2, \ldots, m,
\end{array}\right.
$$

where $\ell_{i}(0, x) \geq 0, \Phi_{i}>0$, and $\bar{\ell}_{i}$ and $\omega_{i}$ satisfy inequality (44).

Remark 4. Comparing the coefficients of the adaptive controllers (31) and (44), it can be concluded that the controllers for global synchronization of the system with time delay are more consumptive.

3.2. Global Synchronization for Systems with Unknown Parameters. In real neural networks, the parameters of the system may be unknown, so it is difficult to achieve synchronization by the conventional adaptive control schemes. In the following text, adaptive controllers with the dynamic compensators are introduced.

Here, suppose that self connection weight $c_{i}$ and input $I_{i}$ in the drive system (15) are unknown. Then, the IVP of the drive system (15) can be written as

$$
\left\{\begin{array}{c}
\frac{\partial^{\alpha} u_{i}(t, x)}{\partial t^{\alpha}}=\sum_{q=1}^{n} d_{i q} \Delta u_{i}(t, x)-c_{i} u_{i}(t, x) \\
+\sum_{j=1}^{m}\left\langle\widetilde{a}_{i j}, \tilde{f}_{1 j}\right\rangle(t, x)+\sum_{j=1}^{m}\left\langle\widetilde{b}_{i j}, \tilde{g}_{1 j}\right\rangle(t-\tau, x)+I_{i}, \\
u_{i}(t, x)=0, \quad t \in[-\tau, \infty), x \in \partial \Omega, \\
u_{i}(s, x)=\varphi_{0 i}(s, x), \quad t \in[-\tau, 0], x \in \Omega .
\end{array}\right.
$$

The IVP of the response system (19) can be shown as

$$
\left\{\begin{array}{l}
\frac{\partial^{\alpha} v_{i}(t, x)}{\partial t^{\alpha}}=\sum_{q=1}^{n} d_{i q} \Delta v_{i}(t, x)-\widehat{c}_{i}(t, x) v_{i}(t, x)+\sum_{j=1}^{m}\left\langle\widetilde{a}_{i j}, \widetilde{f}_{2 j}\right\rangle \\
(t, x)+\sum_{j=1}^{m}\left\langle\widetilde{b}_{i j}, \widetilde{g}_{2 j}\right\rangle(t-\tau, x)+\widehat{I}_{i}(t, x)+\kappa_{i}(t, x), \\
v_{i}(t, x)=0, \quad t \in[-\tau, \infty), x \in \partial \Omega, \\
v_{i}(s, x)=\Phi_{0 i}(s, x), \quad t \in[-\tau, 0], x \in \Omega,
\end{array}\right.
$$

where $i, j=1,2, . ., m$ and $\widehat{c}_{i}(t, x)$ and $\widehat{I}_{i}(t, x)$ are treated as the compensation of $c_{i}$ and $I_{i}$.

Then, for $i, j=1,2, \ldots, m$, the IVP of the synchronization error can be described as

$$
\left\{\begin{array}{l}
\frac{\partial^{\alpha} e_{i}(t, x)}{\partial t^{\alpha}}=-\left(\widehat{c}_{i}(t, x)-c_{i}\right) v_{i}(t, x)-c_{i} e_{i}(t, x)+\sum_{j=1}^{m}\left\langle\widetilde{a}_{i j}, \bar{f}_{j}\right\rangle \\
(t, x)+\sum_{j=1}^{m}\left\langle b_{i j}, \bar{g}_{j}\right\rangle(t-\tau, x)+\left(\widehat{I}_{i}(t, x)-I_{i}\right)+\kappa_{i}(t, x), \\
e_{i}(s, x)=0, \quad s \in[-\tau, \infty), x \in \partial \Omega, \\
e_{i}(s, x)=\bar{\varphi}_{0 i}(s, x), \quad s \in[-\tau, 0], x \in \Omega .
\end{array}\right.
$$

Assumption 3. There are positive constants $p_{i}, q_{i}$, and $\bar{g}_{i}$ such that

$$
\begin{array}{rr}
\sup _{\alpha_{i} \in \overline{\mathrm{co}}\left[f_{i}(\mu)\right], \beta_{i} \in \overline{\mathrm{co}}\left[f_{i}(\vartheta)\right]}\left|\alpha_{i}-\beta_{i}\right| \leq p_{i}\left|\mu_{i}-\vartheta_{i}\right|+q_{i}, & \forall \mu, \vartheta \in \mathbb{R}, \\
& g_{i}(\theta) \leq \bar{g}_{i},
\end{array}
$$

where $i=1,2, \ldots, m, \theta \in \mathbb{R}$, and the notation $\overline{c o}\left[f_{i}(\theta)\right]$ represents $\left[\min \left\{f_{j}^{-}(\theta), f_{j}^{+}(\theta)\right\}, \max \left\{f_{j}^{-}(\theta), f_{j}^{+}(\theta)\right\}\right]$.

Theorem 2. Under Assumption 1 and Assumption 3, the global asymptotic synchronization between (46) and (47) can realized based on the following adaptive controllers:

$$
\left\{\begin{array}{l}
\kappa_{i}(t, x)=-\left(\ell_{i}(t, x)+\bar{\ell}_{i}\right) e_{i}(t, x)-\omega_{i} \operatorname{sgn}\left(e_{i}(t, x)\right), \\
D^{\alpha} \ell_{i}(t, x)=\emptyset_{i} e_{i}^{2}(t, x), \\
D^{\alpha} \widehat{c}_{i}(t, x)=\varrho_{i} e_{i}(t, x) v_{i}(t, x), \\
D^{\alpha} \widehat{I}_{i}(t, x)=-v_{i} e_{i}(t, x),
\end{array}\right.
$$

where $i=1,2, \ldots, m, \ell_{i}(0, x), \varrho_{i}, \varrho_{i}$, and $v_{i}$ are positive constants, and $\bar{\ell}_{i}$ and $\omega_{i}$ are constants which satisfy the following inequalities:

$$
\begin{aligned}
& \bar{\ell}_{i}>\frac{1}{2} \widetilde{\sigma}_{i}>0, \\
& \omega_{i}>\frac{1}{2} \widetilde{\sigma}_{i}^{*},
\end{aligned}
$$

with $\widetilde{\sigma}_{i}=-2\left[c_{i}+\sum_{q=1}^{n}\left(d_{i q} / l_{q}^{2}\right)\right]+\sum_{j=1}^{m}\left(a_{i j}^{\max } p_{j}+a_{j i}^{\max } p_{i}\right)$ and $\tilde{\sigma}_{i}^{*}=2 \sum_{j=1}^{m}\left(a_{i j}^{\max } q_{j}+b_{i j}^{\max } \bar{g}_{j}\right)$.

Proof. Employ the following auxiliary function:

$$
V(t)=V_{1}(t)+V_{2}(t)+V_{3}(t),
$$

where 


$$
\begin{aligned}
& V_{1}(t)=\int_{\Omega} \frac{1}{2} \sum_{i=1}^{m} e_{i}^{2}(t, x) \mathrm{d} x, \\
& V_{2}(t)=\int_{\Omega} \frac{1}{2 \varrho_{i}} \sum_{i=1}^{m}\left(\widehat{c}_{i}(t, x)-c_{i}\right)^{2} \mathrm{~d} x, \\
& V_{3}(t)=\int_{\Omega} \frac{1}{2 \nu_{i}} \sum_{i=1}^{m}\left(\widehat{I}_{i}(t, x)-I_{i}\right)^{2} \mathrm{~d} x .
\end{aligned}
$$

Similarly to the proof of Theorem 1, one obtains

$$
\begin{aligned}
D^{\alpha} V_{1}(t) \leq & \sum_{i=1}^{m} \frac{1}{2} \widetilde{\sigma}_{i} \int_{\Omega} e_{i}^{2}(t, x) \mathrm{d} x \\
& +\sum_{i=1}^{m} \frac{1}{2} \widetilde{\sigma}_{i}^{*} \int_{\Omega}\left|e_{i}(t, x)\right| \mathrm{d} x \\
& +\sum_{i=1}^{m} \int_{\Omega}\left(\widehat{I}_{i}(t, x)-I_{i}\right) e_{i}(t, x) \mathrm{d} x \\
& -\sum_{i=1}^{m} \int_{\Omega}\left(\widehat{c}_{i}(t, x)-c_{i}\right) v_{i}(t, x) e_{i}(t, x) \mathrm{d} x \\
& +\sum_{i=1}^{m} \int_{\Omega} e_{i}(t, x) \kappa_{i}(t, x) \mathrm{d} x, \\
D^{\alpha} V_{3}(t) \leq & -\sum_{i=1}^{m} \int_{\Omega} e_{i}(t, x)\left(\widehat{I}_{i}(t, x)-I_{i}\right) \mathrm{d} x, \\
D^{\alpha} V_{2}(t) \leq & \sum_{i=1}^{m} \int_{\Omega} e_{i}(t, x)\left(\widehat{c}_{i}(t, x)-c_{i}\right) v_{i}(t, x) \mathrm{d} x .
\end{aligned}
$$

Since $\ell_{i}$ and $\omega_{i}$ fulfill condition (51), it follows

$$
D_{t}^{\alpha} V(t) \leq-\lambda_{2} V_{1}(t)
$$

where $\lambda_{2}=\sum_{i=1}^{m}\left(2 \bar{\ell}_{i}-\tilde{\sigma}_{i}\right)$.

By Lemma 5 , it can be concluded that

$$
V(t)-V(0) \leq-\frac{\lambda}{\Gamma(\alpha)} \int_{0}^{t}(t-\tau)^{\alpha-1} V_{1}(\tau) \mathrm{d} \tau .
$$

According to the definition of $V_{1}$, thus

$V_{1}(t) \leq V(t) \leq V(0)-\frac{\lambda}{\Gamma(\alpha)} \int_{0}^{t}(t-\tau)^{\alpha-1} V_{1}(\tau) \mathrm{d} \tau$.

By Lemma 7, it reveals that

$$
\begin{aligned}
V_{1}(t) & \leq V(0) \exp \left(-\frac{\lambda}{\Gamma(\alpha)} \int_{0}^{t}(t-\tau)^{\alpha-1} \mathrm{~d} \tau\right) \\
& =V(0) \exp \left(-\frac{\lambda}{\Gamma(\alpha+1)} t^{\alpha}\right) .
\end{aligned}
$$

Therefore,

$$
\lim _{t \rightarrow \infty} V_{1}(t)=\lim _{t \rightarrow \infty} \int_{\Omega} \frac{1}{2} \sum_{i=1}^{m} e_{i}^{2}(t, x) \mathrm{d} x=0 .
$$

From (59), it is easy to see that the global synchronization between (46) and (47) can be realized.

The proof of Theorem 2 completes.

Remark 5. The result obtained in Theorem 2 is more practical for considering the situation where the parameters of the drive system (24) are unknown. Through the proof of Theorem 2, it can be asserted that the dynamical compensator is necessary for the unknown system. If the reactiondiffusion in space $\Omega$ disappears, the error system (48) can be converted to

$$
\left\{\begin{array}{l}
D^{\alpha} e_{i}(t)=-\left(\widehat{c}_{i}(t)-c_{i}\right) v_{i}(t)-c_{i} e_{i}(t)+\sum_{j=1}^{m}\left\langle\widetilde{a}_{i j}, \bar{f}_{j}\right\rangle(t) \\
+\sum_{j=1}^{m}\left\langle b_{i j}, \bar{g}_{j}\right\rangle(t-\tau)+\left(\widehat{I}_{i}(t)-I_{i}\right)+\kappa_{i}(t), \\
e_{i}(s)=\bar{\varphi}_{0 i}(s), \quad s \in[-\tau, 0] .
\end{array}\right.
$$

Corollary 3. Under Assumption 1 and Assumption 3, the global synchronization between (46) and (47) can be achieved based on the following adaptive controllers:

$$
\left\{\begin{array}{l}
\kappa_{i}(t)=-\left(\ell_{i}(t)+\bar{\ell}_{i}\right) e_{i}(t)-\omega_{i} \operatorname{sgn}\left(e_{i}(t)\right) \\
D^{\alpha} \ell_{i}(t)=\Phi_{i} e_{i}^{2}(t) \\
D^{\alpha} \widehat{c}_{i}(t)=v_{i} e_{i}(t) \\
D^{\alpha} \widehat{I}_{i}(t)=-\varrho_{i} e_{i}(t) \\
i=1,2, \ldots, m
\end{array}\right.
$$

where $\ell_{i}(0) \geq 0, \Phi_{i}>0, \bar{\ell}_{i}>(1 / 2) \widetilde{\sigma}_{i}>0, \omega_{i}>(1 / 2) \widetilde{\sigma}_{i}^{*}$, and $\widetilde{\sigma}_{i}=-2 c_{i}+\left(\sum_{j=1}^{m} a_{i j}^{\max } p_{j}+\sum_{j=1}^{m} a_{j i}^{\max } p_{i}\right)$.

Suppose that there is no time delay $\tau$ in the course of communication; then, the error system (48) can be changed into

$$
\left\{\begin{array}{l}
\frac{\partial^{\alpha} e_{i}(t, x)}{\partial t^{\alpha}}=-\left(\widehat{c}_{i}(t, x)-c_{i}\right) v_{i}(t, x)-c_{i} e_{i}(t, x) \\
+\sum_{j=1}^{m}\left\langle\widetilde{a}_{i j}, \bar{f}_{j}\right\rangle(t, x)+\left(\widehat{I}_{i}(t, x)-I_{i}\right)+\kappa_{i}(t, x), \\
e_{i}(s, x)=0, \quad s \in[0, \infty), x \in \partial \Omega, \\
e_{i}(s, x)=\bar{\varphi}_{0 i}(s, x), \quad s=0, x \in \Omega .
\end{array}\right.
$$

Corollary 4. Under Assumption 1 and Assumption 3, (46) and (47) can achieve global synchronization based on the following adaptive controllers: 


$$
\left\{\begin{array}{l}
\kappa_{i}(t, x)=-\left(\bar{\ell}_{i}(t, x)+\ell_{i}\right) e_{i}(t, x)-\omega_{i} \operatorname{sgn}\left(e_{i}(t, x)\right), \\
D^{\alpha} \ell_{i}(t, x)=\emptyset_{i} e_{i}^{2}(t, x), \\
D^{\alpha} \widehat{c}_{i}(t, x)=v_{i} e_{i}(t, x), \\
D^{\alpha} \widehat{I}_{i}(t, x)=-\varrho_{i} e_{i}(t, x), \\
i=1,2, \ldots, m,
\end{array}\right.
$$

where $\ell_{i}(0, x) \geq 0, \Phi_{i}>0, \bar{\ell}_{i}>(1 / 2) \breve{\sigma}_{i}>0, \omega_{i}>(1 / 2) \vec{\sigma}_{i}^{*}$, and $\breve{\sigma}_{i}^{*}=2 \sum_{j=1}^{m} a_{i j}^{\max } q_{j}$.

\section{Numerical Simulations}

Several numerical simulations are presented to illustrate the main results. The first example is used to validate Theorem 1. The second one is used to text Theorem 2. Now, let us consider the $2 \mathrm{D}(q=1)$ reaction-diffusion FMNNs with time delay and zero Direclet boundary values. Here, $m=2$, $n=1$, and $\Omega=[-2,2]$.

Example 1. Consider error system (26) with such parameters:

$$
\begin{aligned}
& A=\left(\begin{array}{cc}
a_{11} & -0.02 \\
0.13 & -0.01
\end{array}\right), \\
& B=\left(\begin{array}{cc}
0.35 & b_{12} \\
0.36 & 0.37
\end{array}\right), \\
& C=\left(\begin{array}{cc}
0.35 & 0 \\
0 & 0.23
\end{array}\right), \\
& I=\left(\begin{array}{cc}
0.60 & 0 \\
0 & 0.40
\end{array}\right),
\end{aligned}
$$

where the memristive coefficients are defined by

$$
\begin{aligned}
& a_{11}= \begin{cases}0.19, & \delta<1, \\
0.26, & \delta=1, \\
0.55, & \delta>1,\end{cases} \\
& b_{12}= \begin{cases}-0.13, & \delta<1, \\
-0.17, & \delta=1, \\
-0.24, & \delta>1 .\end{cases}
\end{aligned}
$$

Suppose that time delay $\tau=1.1$ and discontinuous activation functions are $f(\delta)=g(\delta)=0.98 \delta+0.05 \operatorname{sgn}(\delta)$. Let initial values of (24) and (25) be

$$
\begin{aligned}
& \left\{\begin{array}{l}
u_{1}(x, t)=8 \sin \left(-2+x+t^{2}\right), \\
u_{2}(x, t)=6 \sin (-2+x+t),
\end{array}\right. \\
& \left\{\begin{array}{l}
v_{1}(x, t)=-5 \cos (-2+x+t), \\
v_{2}(x, t)=-6 \cos (-2+x+t),
\end{array}\right.
\end{aligned}
$$

where $t \in[-1.1,0]$ and $x \in(-2,2)$. Given the fractional order $\alpha=0.98$ and $d_{11}=d_{21}=0.85$. Under these parameters, the dynamical behaviors of the synchronization errors between (24) and (25) are shown in Figure 1. From the figure, it can be observed that the synchronization errors gradually increase as time goes on. Notice that $f(\delta)$ and $g(\delta)$ have only one discontinuous point 0 , Assumption 1 is valid. According to Assumption 2, we can choose $p_{1}=p_{2}=$ $r_{1}=r_{2}=0.98$ and $q_{1}=q_{2}=s_{1}=s_{2}=0.05$. By calculation, the parameters of controllers are selected as $\bar{\ell}_{1}=0.70>0.39$, $w_{1}=0.06>0.05, \quad \bar{\ell}_{2}=0.60>0.36, \quad w_{2}=0.05>0.04$, $\ell_{1}(0, x)=\ell_{2}(0, x)=0.01>0$, and $\Phi_{1}=\Phi_{2}=0.01>0$. It is easy to verify that condition (9) in Theorem 1 is satisfied. Figure 2 suggests that the synchronization errors asymptotically converge to zero.

Theorem 1 is not only dependent on order but also related with reaction-diffusion coefficients. Figure 3 shows the trajectories of the error system at spatial position $x=55$ with different orders $\alpha=0.6,0.7,0.8,0.9$ when the reactiondiffusion coefficients $d=1.8$. While if the order is fixed $\alpha=0.7$, the trajectories of the error system at spatial position $x=55$ with different reaction-diffusion coefficients $d=0.6,1.4,2.2,3.0$ are shown in Figure 4. From the numerical results, we could note that the error system would achieve the asymptotic stability faster with the higher order. It also holds true with increasing reaction-diffusion coefficient.

Example 2. Consider the error system (48) with the following parameters:

$$
\begin{aligned}
A & =\left(\begin{array}{cc}
a_{11} & -0.95 \\
0.58 & 0.76
\end{array}\right), \\
B & =\left(\begin{array}{cc}
0.22 & b_{12} \\
0.15 & 0.03
\end{array}\right), \\
C & =\left(\begin{array}{cc}
0.58 & 0 \\
0 & 0.49
\end{array}\right), \\
I & =\left(\begin{array}{cc}
0.23 & 0 \\
0 & 0.25
\end{array}\right),
\end{aligned}
$$

where

$$
\begin{aligned}
& a_{11}= \begin{cases}0.19, & \delta<1, \\
0.80, & \delta=1, \\
0.26, & \delta>1,\end{cases} \\
& b_{12}= \begin{cases}0.05, & \delta<1, \\
0.11, & \delta=1, \\
0.06, & \delta>1 .\end{cases}
\end{aligned}
$$

Assume $\alpha=0.98, d_{11}=d_{21}=0.85$, and (47) with unknown parameters $c_{i}$ and $I_{i}$, and initial values of compensators in (47) are given by

$$
\begin{aligned}
\widehat{C}_{0} & =\left(\begin{array}{ll}
1 & 0 \\
0 & 1
\end{array}\right), \\
\widehat{I}_{0} & =\left(\begin{array}{ll}
2 & 0 \\
0 & 2
\end{array}\right) .
\end{aligned}
$$




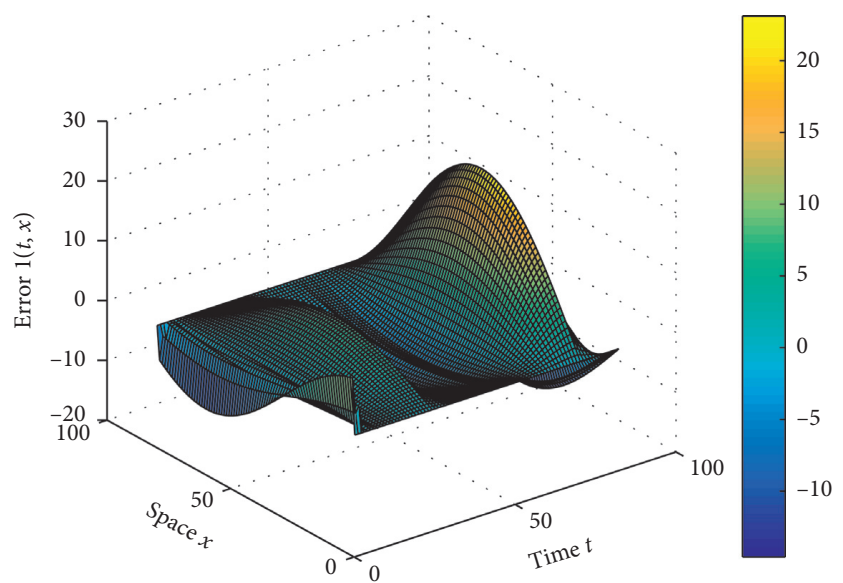

(a)

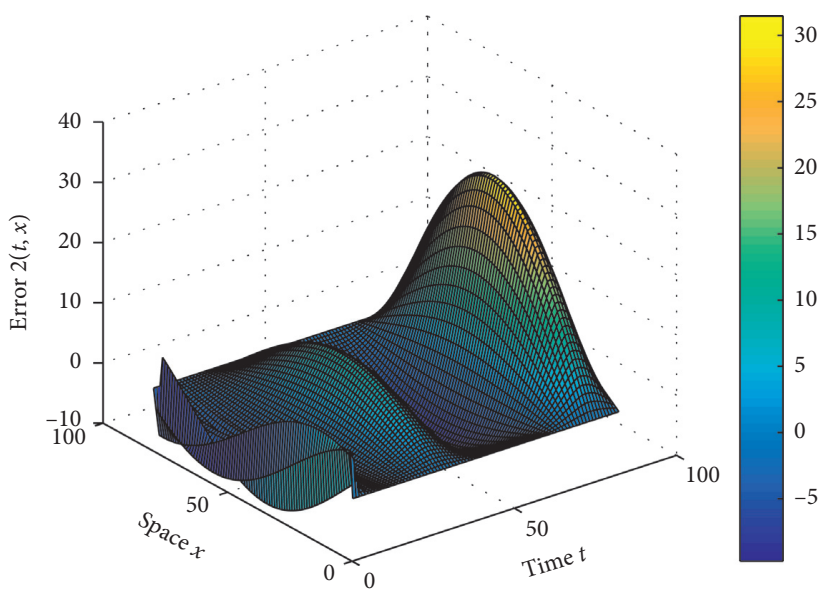

(b)

FIGURE 1: Synchronization errors between systems (24) and (25), with no controller.

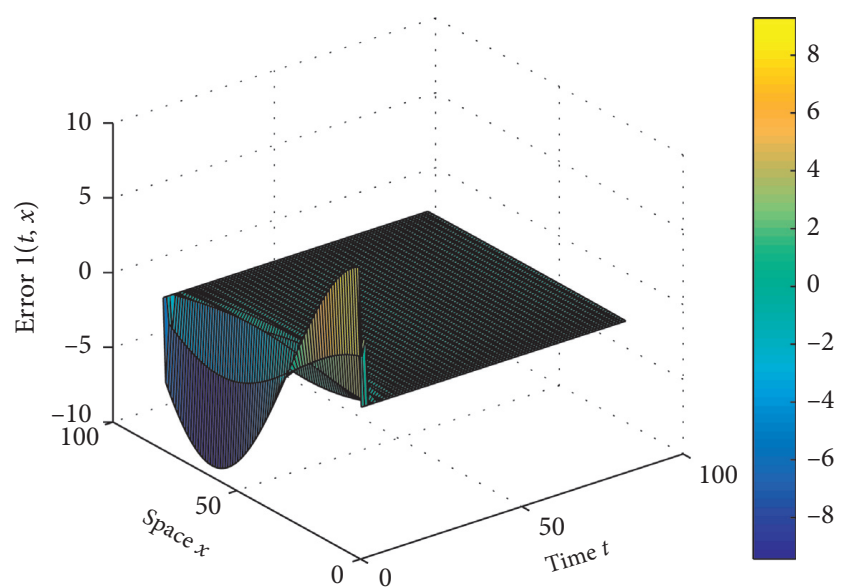

(a)

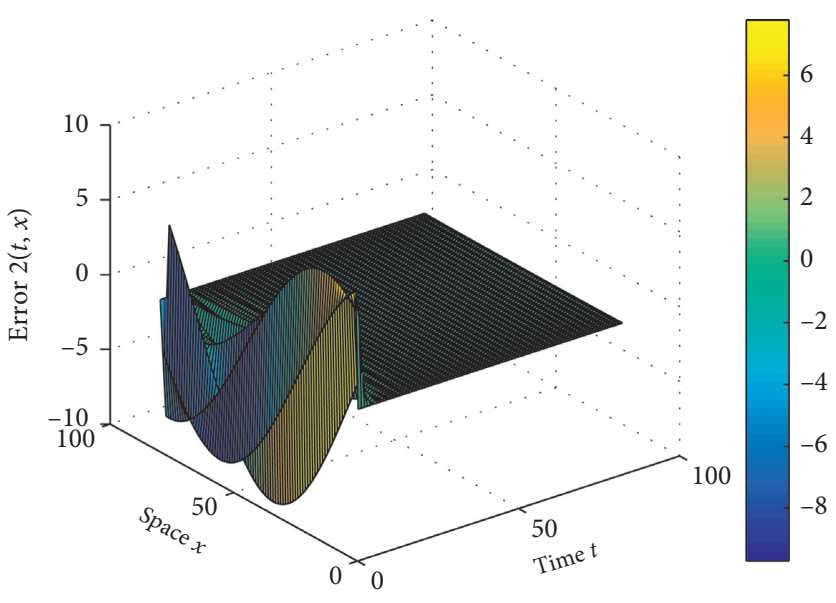

(b)

Figure 2: Synchronization errors between systems (24) and (25), under controller (30).

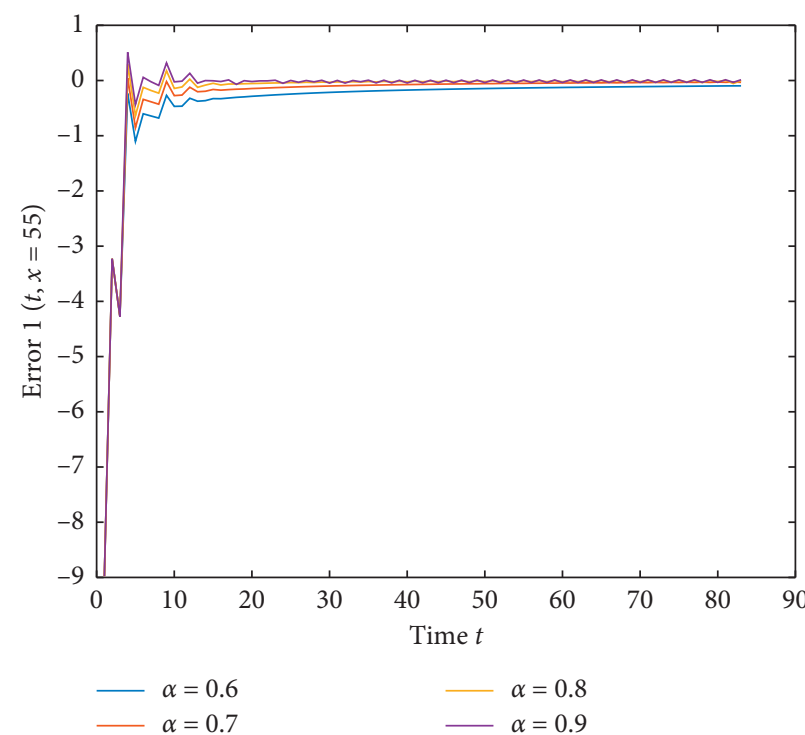

(a)

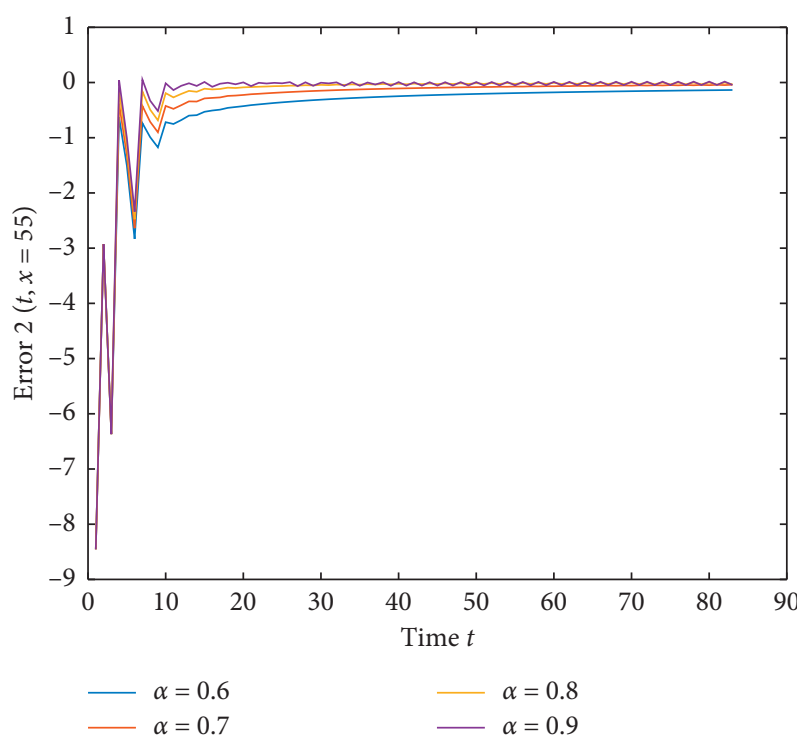

(b)

Figure 3: Trajectories of error system (26) at spatial position $x=55$ under different orders. 


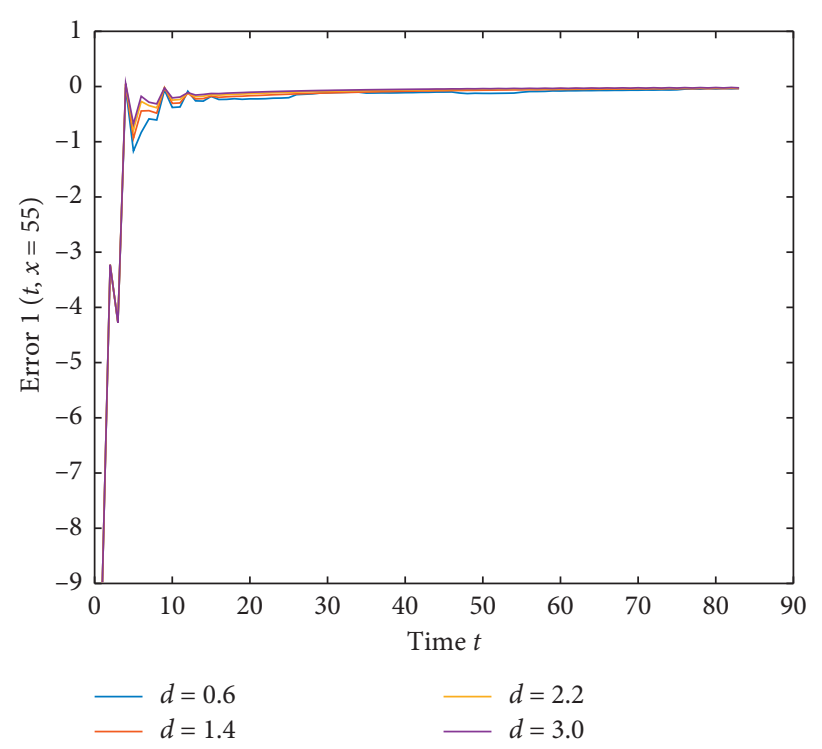

(a)

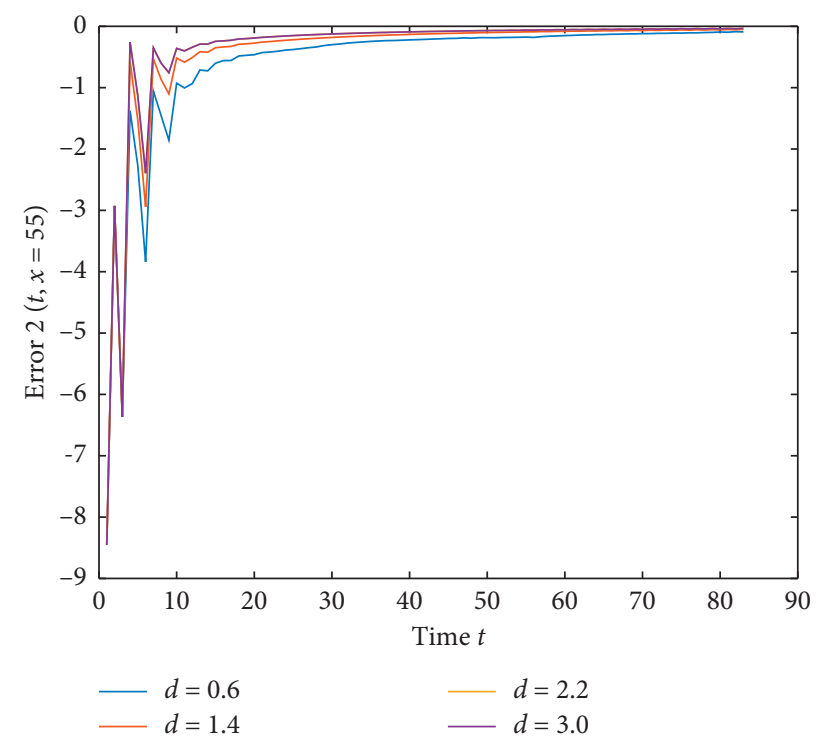

(b)

FIgURE 4: Trajectories of error system (26) at spatial position $x=55$ under different diffusion coefficients.

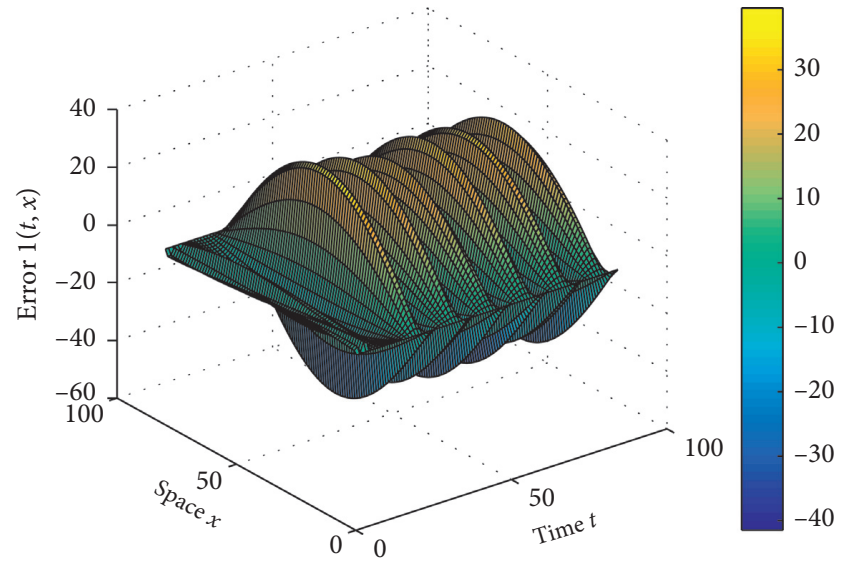

(a)

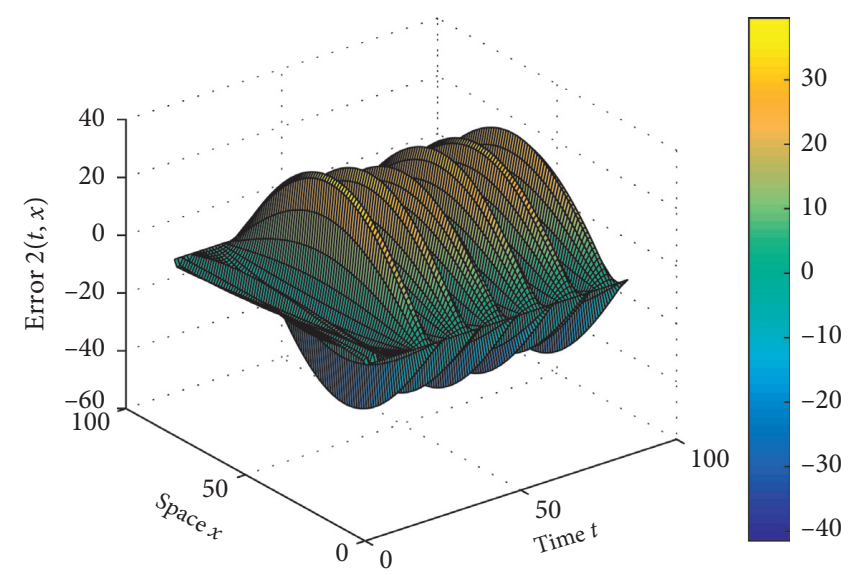

(b)

FIGURE 5: Synchronization errors between systems (46) and (47), with no controller.

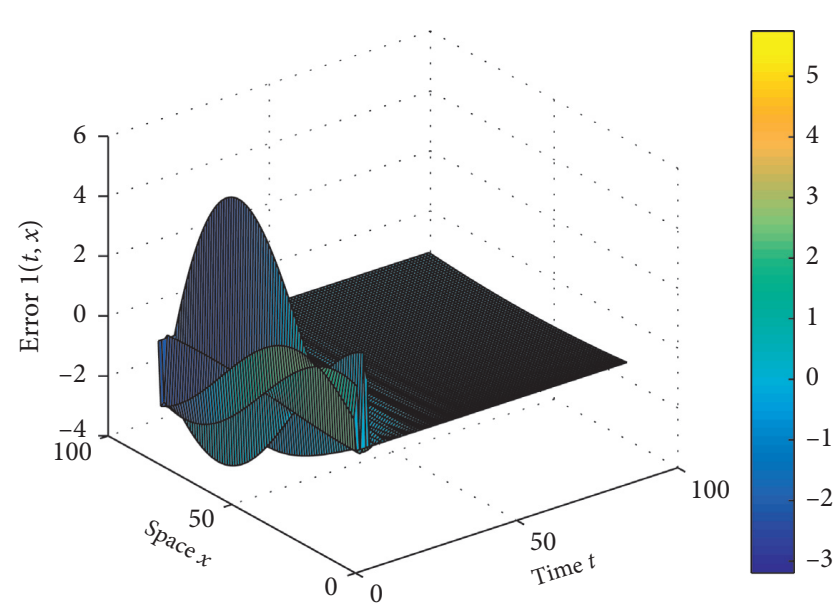

(a)

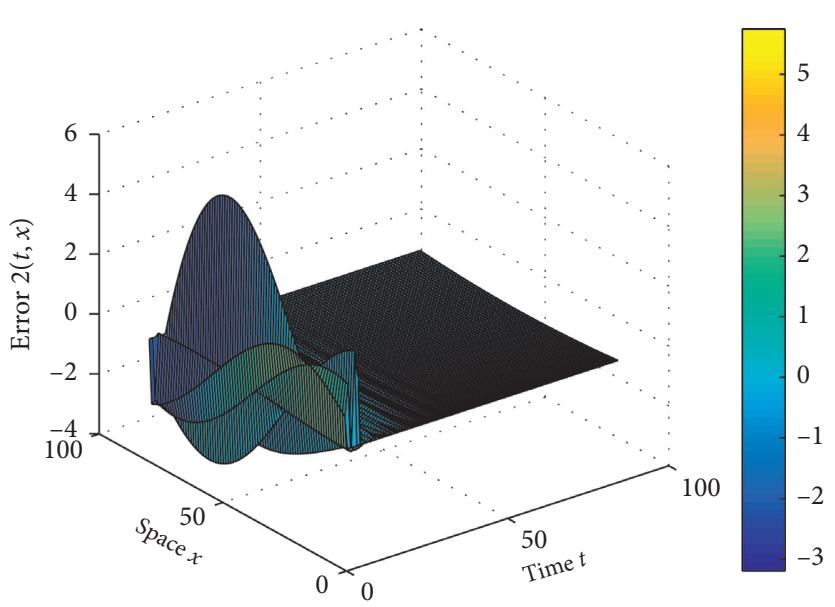

(b)

FIGURE 6: Synchronization errors between systems (46) and (47), with controller (50). 


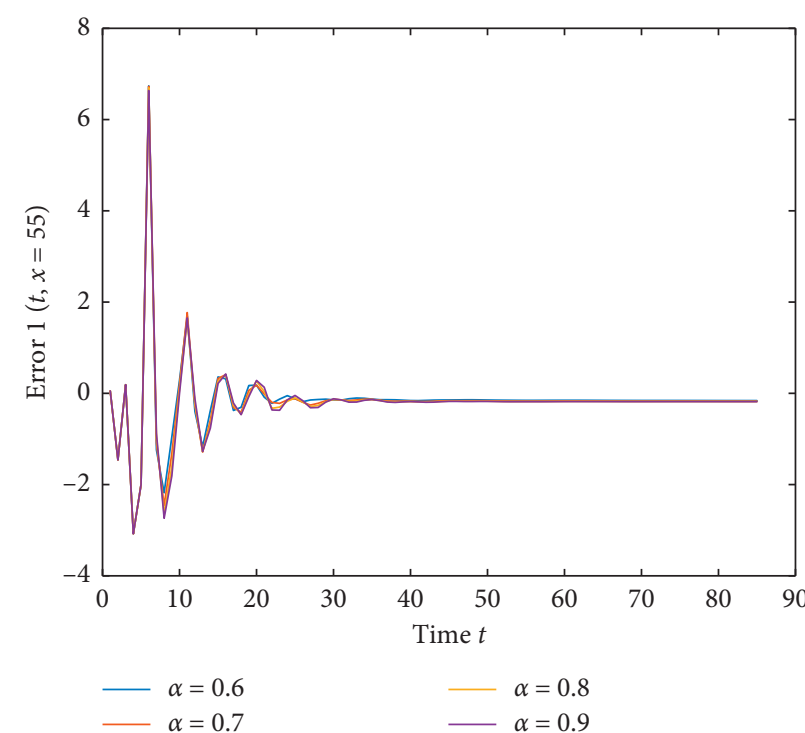

(a)

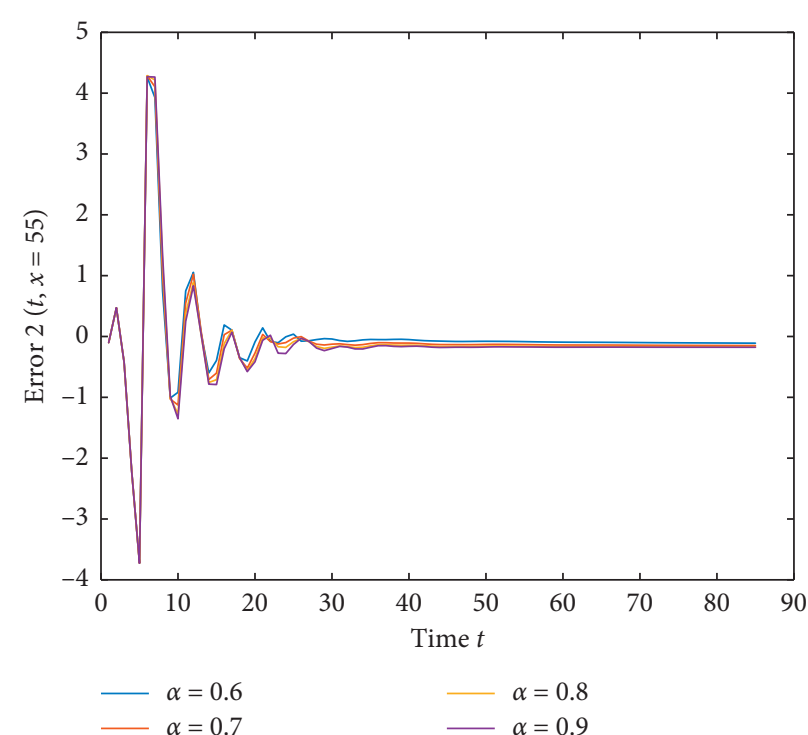

(b)

Figure 7: Trajectories of error system (26) at spatial position $x=55$ under different orders.

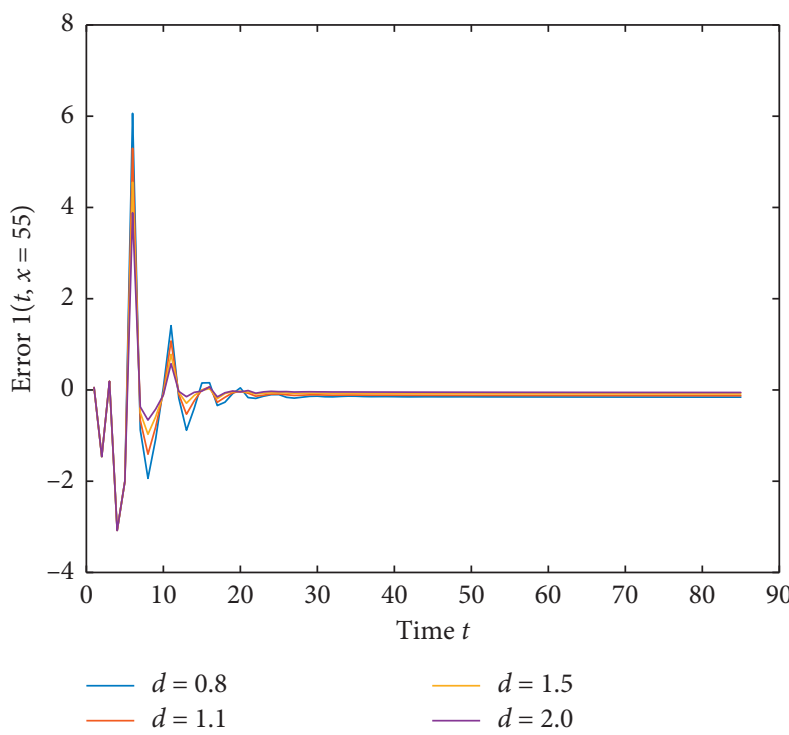

(a)

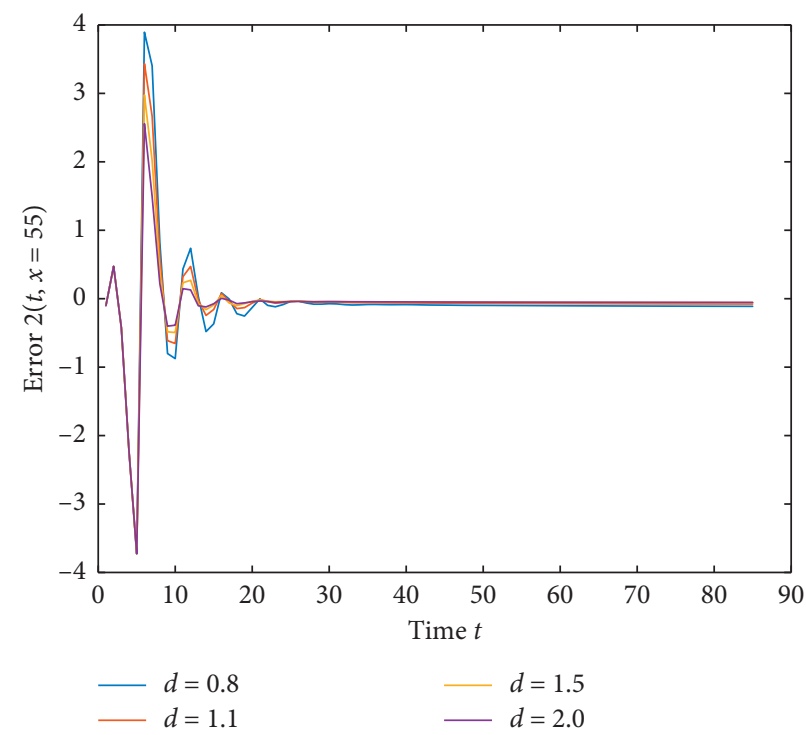

(b)

FIgURE 8: Trajectories of error system (26) at spatial position $x=55$ under different diffusion coefficients.

Activation functions are $f(\delta)=0.98 \delta+0.05 \operatorname{sgn}(\delta)$ and $g(\delta)=0.5 \operatorname{sgn}(\delta)$ and time delay $\tau=3.5$. Initial values of $(46)$ and (47) are chosen as

$$
\begin{aligned}
& \left\{\begin{array}{l}
u_{1}(x, t)=1.7 \sin \left(-2+x+t^{2}\right), \\
u_{2}(x, t)=-2.1 \sin (-2+x+t),
\end{array}\right. \\
& \left\{\begin{array}{l}
v_{1}(x, t)=1.5 \cos (-2+x+8 t), \\
v_{2}(x, t)=-1.9 \cos \left(-2+x+8 t^{2}\right),
\end{array}\right.
\end{aligned}
$$

where $t \in[-3.4,0]$ and $x \in(-2,2)$.
As seen in Figure 5, the error state is instable. Choosing $p_{1}=p_{2}=0.98, q_{1}=q_{2}=0.05$, and $\bar{g}_{1}=\bar{g}_{2}=0.5$, we obtain $\bar{\ell}_{1}=1.10>1.06, w_{1}=0.11>0.10, \bar{\ell}_{2}=1.00>0.97$, and $w_{2}=$ $0.08>0.07 \quad$ and $\quad \ell_{i}(0, x)=0.01>0, \quad \Phi_{i}=0.01>0$, $\varrho_{i}=0.001>0$, and $v_{i}=0.02>0, i=1,2$. Condition (51) in Theorem 2 is content clear. Figure 6 shows that the synchronization errors achieve stability rapidly under the adaptive controller (50).

For the error system (48), under different orders $\alpha=$ $0.6,0.7,0.8,0.9$ with the same reaction-diffusion coefficients $d=0.6$ and different reaction-diffusion coefficients 
$d=0.8,1.1,1.5,2.0$ with the same order $\alpha=0.7$, simulations are carried out. Corresponding error state trajectories are depicted in Figures 7 and 8. It reveals that the asymptotic stability of the system varies with orders and reaction-diffusion coefficients. Compared with the former case, the figures show little differences with different parameters.

\section{Conclusion and Discussion}

The global synchronization problem for reaction-diffusion FMNNs with time delay was emphasized firstly in this paper. Both the known and unknown parameters cases were investigated, separately. Based on fractional comparison theorem and Gronwall-Bellman inequality, two kinds of adaptive control schemes were proposed. Numerical simulations were implemented to examine the effectiveness of the obtained results.

Conventional adaptive controllers have mostly been used for controlling neural networks system, while they cannot guarantee the synchronization of unknown systems. An adaptive controller with a dynamic compensator such as the one described in this paper can eliminate this problem. However, the true values of the unknown parameters could not be obtained. Further research is still in progress to design an adaptive controller with dynamic compensator to achieve the identity of parameters.

\section{Data Availability}

No data were used to support this study.

\section{Conflicts of Interest}

The authors declare that they have no conflicts of interest.

\section{Acknowledgments}

This work was supported by the National Nature Science Foundation of China (no. 61772063) and Beijing Natural Science Foundation (no. Z180005).

\section{References}

[1] A. A. A. Kilbas, H. M. Srivastava, and J. J. Trujillo, Theory and Applications of Fractional Differential Equations, Vol. 204, Elsevier Science Limited, Amsterdam, Netherlands, 2006.

[2] B. B. Mandelbrot and J. A. Wheeler, "The fractal geometry of nature," American Journal of Physics, vol. 51, no. 3, pp. 286-287, 1983.

[3] A. Boroomand and M. B. Menhaj, "Fractional-order hopfield neural networks," in Proceedings of the International Conference on Neural Information Processing, pp. 883-890, Auckland, New Zealand, November 2008.

[4] H. Wei, R. Li, C. Chen, and Z. Tu, "Stability analysis of fractional order complex-valued memristive neural networks with time delays," Neural Processing Letters, vol. 45, no. 2, pp. 379-399, 2017.

[5] X. Yang, C. Li, T. Huang, Q. Song, and X. Chen, "Quasiuniform synchronization of fractional-order memristorbased neural networks with delay," Neurocomputing, vol. 234, pp. 205-215, 2017.
[6] Q. Zou, Q. Jin, and R. Zhang, "Design of fractional order predictive functional control for fractional industrial processes," Chemometrics and Intelligent Laboratory Systems, vol. 152, pp. 34-41, 2016.

[7] P. Arena, R. Caponetto, L. Fortuna, and D. Porto, "Bifurcation and chaos in noninteger order cellular neural networks," International Journal of Bifurcation and Chaos, vol. 8, no. 7, pp. 1527-1539, 1998.

[8] H. Zhang, X.-Y. Wang, and X. H. Lin, "Topology identification and module-phase synchronization of neural network with time delay," IEEE Transactions on Systems, Man, and Cybernetics: Systems, vol. 47, no. 6, pp. 885-892, 2016.

[9] H. L. Trentelman, K. Takaba, and N. Monshizadeh, "Robust synchronization of uncertain linear multi-agent systems," IEEE Transactions on Automatic Control, vol. 58, no. 6, pp. 1511-1523, 2013.

[10] A. Abdurahman, H. Jiang, and Z. Teng, "Finite-time synchronization for memristor-based neural networks with timevarying delays," Neural Networks, vol. 69, pp. 20-28, 2015.

[11] J. Gao, P. Zhu, W. Xiong, J. Cao, and L. Zhang, "Asymptotic synchronization for stochastic memristor-based neural networks with noise disturbance," Journal of the Franklin Institute, vol. 353, no. 13, pp. 3271-3289, 2016.

[12] L. Chen, R. Wu, J. Cao, and J.-B. Liu, "Stability and synchronization of memristor-based fractional-order delayed neural networks," Neural Networks, vol. 71, pp. 37-44, 2015.

[13] T. Hu, Z. He, X. Zhang, and S. Zhong, "Global synchronization of time-invariant uncertainty fractional-order neural networks with time delay," Neurocomputing, vol. 339, pp. 45-58, 2019.

[14] P. Liu, Z. Zeng, and J. Wang, "Global synchronization of coupled fractional-order recurrent neural networks," IEEE Transactions on Neural Networks and Learning Systems, vol. 30, no. 8, pp. 2358-2368, 2019.

[15] T. Hu, X. Zhang, and S. Zhong, "Global asymptotic synchronization of nonidentical fractional-order neural networks," Neurocomputing, vol. 313, pp. 39-46, 2018.

[16] L. Chua, "Memristor-the missing circuit element," IEEE Transactions on Circuit Theory, vol. 18, no. 5, pp. 507-519, 1971.

[17] D. B. Strukov, G. S. Snider, D. R. Stewart, and R. S. Williams, "The missing memristor found," Nature, vol. 453, no. 7191, pp. 80-83, 2008.

[18] G. Velmurugan, R. Rakkiyappan, and J. Cao, "Finite-time synchronization of fractional-order memristor-based neural networks with time delays," Neural Networks, vol. 73, pp. 36-46, 2016.

[19] L. Zhang, Y. Yang, and F. Wang, "Lag synchronization for fractional-order memristive neural networks via period intermittent control," Nonlinear Dynamics, vol. 89, no. 1, pp. 367-381, 2017.

[20] L. Chen, J. Cao, R. Wu, J. A. Tenreiro Machado, A. M. Lopes, and H. Yang, "Stability and synchronization of fractionalorder memristive neural networks with multiple delays," Neural Networks, vol. 94, pp. 76-85, 2017.

[21] L. Chen, T. Huang, J. A. Tenreiro Machado, A. M. Lopes, Y. Chai, and R. Wu, "Delay-dependent criterion for asymptotic stability of a class of fractional-order memristive neural networks with time-varying delays," Neural Networks, vol. 118, pp. 289-299, 2019.

[22] I. D. Landau, R. Lozano, M. M'Saad et al., Adaptive Control, Vol. 51, Springer, Berlin, Germany, 1998.

[23] S. Chang and T. Peng, "Adaptive guaranteed cost control of systems with uncertain parameters," IEEE Transactions on Automatic Control, vol. 17, no. 4, pp. 474-483, 1972. 
[24] C. Wang, M. Liang, and Y. Chai, "An adaptive control of fractional-order nonlinear uncertain systems with input saturation," Complexity, vol. 2019, Article ID 5643298, 17 pages, 2019.

[25] H. Liu, Y. Pan, and J. Cao, "Composite learning adaptive dynamic surface control of fractional-order nonlinear systems," IEEE Transactions on Cybernetics, vol. 50, no. 6, pp. 2557-2567.

[26] M. F. Arevalo Castiblanco, D. Tellez Castro, J. Sofrony, and E. Mojica Nava, "Adaptive control for unknown heterogeneous vehicles synchronization with unstructured uncertainty," in Proceeding of the 2019 IEEE 4th Colombian Conference on Automatic Control (CCAC), pp. 1-6, Medellín, Colombia, October 2019.

[27] Z. Xiong, S. Qu, and J. Luo, "Adaptive multi-switching synchronization of high-order memristor-based hyperchaotic system with unknown parameters and its application in secure communication," Complexity, vol. 2019, Article ID 3827201, 18 pages, 2019.

[28] L. Chen, R. Wu, Z. Chu, Y. He, and L. Yin, "Pinning synchronization of fractional-order delayed complex networks with non-delayed and delayed couplings," International Journal of Control, vol. 90, no. 6, pp. 1245-1255, 2017.

[29] F. Wang, Z. Zheng, and Y. Yang, "Synchronization of complex dynamical networks with hybrid time delay under eventtriggered control: the threshold function method," Complexity, vol. 2019, Article ID 7348572, 17 pages, 2019.

[30] Z. Yang, W. Zhou, and T. Huang, "Input-to-state stability of delayed reaction-diffusion neural networks with impulsive effects," Neurocomputing, vol. 333, pp. 261-272, 2019.

[31] X. Lu, W.-H. Chen, Z. Ruan, and T. Huang, "A new method for global stability analysis of delayed reaction-diffusion neural networks," Neurocomputing, vol. 317, pp. 127-136, 2018.

[32] I. Stamova and G. Stamov, "Mittag-Leffler synchronization of fractional neural networks with time-varying delays and reaction-diffusion terms using impulsive and linear controllers," Neural Networks, vol. 96, pp. 22-32, 2017.

[33] I. Stamova and S. Simeonov, "Delayed reaction-diffusion cellular neural networks of fractional order: mittag-leffler stability and synchronization," Journal of Computational and Nonlinear Dynamics, vol. 13, no. 1, Article ID 011015, 2018.

[34] H. Liu, S. Li, H. Wang, and Y. Sun, "Adaptive fuzzy control for a class of unknown fractional-order neural networks subject to input nonlinearities and dead-zones," Information Sciences, vol. 454-455, pp. 30-45, 2018.

[35] S. Li, X. Peng, Y. Tang, and Y. Shi, "Finite-time synchronization of time-delayed neural networks with unknown parameters via adaptive control," Neurocomputing, vol. 308, pp. 65-74, 2018.

[36] Y. Gu, Y. Yu, and H. Wang, "Synchronization-based parameter estimation of fractional-order neural networks," Physica A: Statistical Mechanics and Its Applications, vol. 483, pp. 351-361, 2017.

[37] W. F. Ames, "Mathematics in science and engineering," in Fractional Differential Equations, I. Podlubny, Ed., Vol. 198, Elsevier, Amsterdam, Netherlands, 1999.

[38] H. Wang, Y. Yu, G. Wen, S. Zhang, and J. Yu, "Global stability analysis of fractional-order hopfield neural networks with time delay," Neurocomputing, vol. 154, pp. 15-23, 2015.

[39] Z. Ouyang, "Existence and uniqueness of the solutions for a class of nonlinear fractional order partial differential equations with delay," Computers \& Mathematics with Applications, vol. 61, no. 4, pp. 860-870, 2011.
[40] W. Ma, C. Li, Y. Wu, and Y. Wu, "Adaptive synchronization of fractional neural networks with unknown parameters and time delays," Entropy, vol. 16, no. 12, pp. 6286-6299, 2014.

[41] J. G. Lu, "Global exponential stability and periodicity of reaction-diffusion delayed recurrent neural networks with Dirichlet boundary conditions," Chaos, Solitons \& Fractals, vol. 35, no. 1, pp. 116-125, 2008.

[42] J.-J. E. SlotineW. Li et al., Applied Nonlinear Control, Vol. 199, Prentice Hall, Englewood Cliffs, NJ, USA, 1991.

[43] P. Phung and L. Truong, "On a fractional differential inclusion with integral boundary conditions in banach space," Fractional Calculus and Applied Analysis, vol. 16, no. 3, pp. 538-558, 2013.

[44] M. Benchohra, J. Henderson, S. K. Ntouyas, and A. Ouahab, "Existence results for fractional functional differential inclusions with infinite delay and applications to control theory," Fractional Calculus and Applied Analysis, vol. 11, no. 1, pp. 35-56, 2008.

[45] Z. Tu, N. Ding, L. Li, Y. Feng, L. Zou, and W. Zhang, "Adaptive synchronization of memristive neural networks with time-varying delays and reaction-diffusion term," Applied Mathematics and Computation, vol. 311, pp. 118-128, 2017. 